Monatsschrift f. Geburtshülfe u. Gynäkologie 1931;88:I-II

\title{
Contents, Vol. 88, No. 3-4, 1931
}

Inhaltsverzeichnis. Original-Arbeiten.

Seite

Schilddrứsenbehandluńg vor und während der Tragperiode und deren Eínfluß auî die

Nachkommenschaft. Von Professor Dr. F. Gudernatsch-New York 161

Ein Beitrag zur Leberschädigung bei Eklampsie, Eklampsie ohne Krämpfe und bei Hyperemesís.

Von Dr. Karl Rosen-loecher-¥vddMxxri am Main 163

III. Über die Anwendung des Pernocton bei Eklampsie. Von

H. Goec.ke-Köin 170

IV. Klinik und Cliemie des Pernoctons in der Geburtshilfe und

Gynäkologie. Von F. Fretwurst und F. B, Ruder-Hamburg 179

V. Blutungen bei Frauen über fünfzig Jahre. Von Dr. K Tietse

und cand. med. Clemens Mayer-K\t $\backslash 185$

VI. Röntgenkontrollen íubarer Sterilisationen. Von H.Fuchs und

Erich C. Lork 199

VII. Pyosalpinx und Salpingitis und ihre Behandlung in it Prolan.

Von Medizinalrat Or. H. Monlag-Bochum 212

VIII. Torsion einesgroßen Uterusmyom und Wiederaufplatzen der

Bauchwunde. Von Dr. Frits Heinsius-Bzúm 224

IX. Über postoperative Thrombose.Von Professor W. Lindemann-

Halle 229

X. Eiufluß der Arbeit in der Tabakindustrie auf die Geschlechts-sphäre der Arbeiterin. Von

Professor M. Myalobeli-TúWs . 237

Monatsschrift f. Geburtshiilfe u. Gynäkologie. Bd. LXXXVIII Heft 3/4. 11

Seite

Grenzgebieie der Geburtshilfe und Gynakologie.

Kurzer Bericht fiber Lebererkrankungen, Uropoetisches System

und Nierenerkrankungen. Von Priv.-Doz. Dr. Werner Bicken-

bach-Bonn 248

Vereins- und Liíeraiurbeilage.

Original-Sitzungsberichte aus geburtshilflich-gynäkologischen Gesellschaften.

Gynäkologische Gesellschaft zu Breslau. Sitzuสg vom 20. Januar

1931258

Gesellschaft für Geburtshilfe und Gynakologie zu Berlin. Sitzungen

vom 28. November 1930, 23. und 30. Januar und 13. Fe-

bruar $1931 \quad 268$

Bayerische Gesellschaft für Geburtshilfe und Trauenheilkunde.

Sitzung vom 23. Februar 1930 in München (Schluß) . . .285

Niederrheínísch-westfälische Gesellschaft für Gynakologie und

Geburtshilfe. 94. Sitzung am 29. November 1930 zu Düsseldorf 320 
Geburtshilflich-gynäkologische Gesellschaft in Wien. Sitzung vom 10. Februar $1931 \quad 332$ 